\title{
Gravitational Waves in Massive Gravity Theories: Waveforms, Fluxes, and Constraints from Extreme-Mass-Ratio Mergers
}

\author{
Vitor Cardoso, ${ }^{1,2, *}$ Gonçalo Castro, ${ }^{1, \dagger}$ and Andrea Maselli ${ }^{1,3, *}$ \\ ${ }^{1}$ Centro de Astrofísica e Gravitação-CENTRA, Departamento de Física, Instituto Superior Técnico-IST, \\ Universidade de Lisboa-UL, Av. Rovisco Pais 1, 1049-001 Lisboa, Portugal \\ ${ }^{2}$ Theoretical Physics Department, CERN 1 Esplanade des Particules, CH-1211 Geneva 23, Switzerland \\ ${ }^{3}$ Dipartimento di Fisica, "Sapienza" Università di Roma, Piazzale Aldo Moro 5, 00185 Roma, Italy
}

(Received 17 July 2018; revised manuscript received 5 November 2018; published 17 December 2018)

Is the graviton massless? This problem was addressed in the literature at a phenomenological level, using modified dispersion relations for gravitational waves, in linearized calculations around flat space. Here, we perform a detailed analysis of the gravitational waveform produced when a small particle plunges or inspirals into a large nonspinning black hole. Our results should presumably also describe the gravitational collapse to black holes and explosive events such as supernovae. In the context of a theory with massive gravitons and screening, merging objects up to $1 \mathrm{Gpc}$ away or collapsing stars in the nearby galaxy may be used to constrain the mass of the graviton to be smaller than $\sim 10^{-23} \mathrm{eV}$, with low-frequency detectors. Our results suggest that the absence of dipolar gravitational waves from black hole binaries may be used to rule out entirely such theories.

DOI: 10.1103/PhysRevLett.121.251103

Introduction.-General relativity (GR) is special and unique in a very precise mathematical sense [1,2]. Nevertheless, several arguments suggest that such an elegant theory cannot easily accommodate neither the ultraviolet nor the infrared description of the universe. Simultaneously, observations of large-scale phenomena indicate that either the matter sector or the gravitational interaction requires a better understanding. In other words, extensions of GR are welcome. One of the possible extensions draws inspiration from the standard model of particle physics and consists in allowing for a massive graviton [2-5].

Bounds on such theories can be imposed via gravitationalwave $(\mathrm{GW})$ emission and propagation mechanisms. These include the following: (i) Modified dispersion relations for GWs, assuming that their generation is as in GR [6-8]. (ii) The spin down of black holes (BHs), caused by superradiant instabilities [9]. (iii) Changes in the orbital period of binary pulsars, caused by a different energy flux [10].

Other mechanisms may also help in bounding the graviton mass, such as modifications of the GW memory effect [11]. There are no constraints using directly the measured properties of GWs, without any assumption on the production mechanism. Our main concern here is precisely to compute the gravitational waveform and fluxes

Published by the American Physical Society under the terms of the Creative Commons Attribution 4.0 International license. Further distribution of this work must maintain attribution to the author(s) and the published article's title, journal citation, and DOI. from the merger of two compact objects, using the strongfield regime of massive gravity theories. We consider the ghost-free theory describing two interacting spin-2 fields described in detail in the Supplemental Material [12].

Following all observational evidence thus far, we consider only BHs which are as similar as possible to those in GR; in particular, we study Schwarzschild BHs which are also exact solutions of massive bi-gravity theories. We focus on the truly unique features of massive gravity theories: the extra polarizations with respect to GR and their signatures on the GW emission. We thus consider mergers of extreme-mass ratio objects in which the massive one is a Schwarzschild BH. We will show that the extra degrees of freedom give rise to substantially different GW signals, even when the underlying backgrounds are exactly the same.

Throughout this Letter, we use geometrized units, in which $G=c=1$.

Formalism and master equations. - In our framework, a small point particle is orbiting, or merging with, a massive Schwarzschild BH of mass $M$. This system may model the merger of a neutron star with a stellar-mass or a supermassive $\mathrm{BH}$, but it may well describe qualitatively the merger of two equal-mass BHs as well. In fact, the lesson from the two-body problem in GR is that perturbation theory is able to account for this process even at a quantitative level [17]. The point particle moves on a spacetime geodesic $y_{p}^{\mu}(\tau)=\left(t_{p}(\tau), r_{p}(\tau), \theta_{p}(\tau), \varphi_{p}(\tau)\right)$, with $\tau$ being the test body proper time. The particle is taken to be pointlike and described by the stress-energy tensor 


$$
T^{\mu \nu}=m_{p} \int(-g)^{1 / 2} u^{\mu} u^{\nu} \delta^{(4)}\left(x^{\beta}-y_{p}^{\beta}\right) d \tau,
$$

where $m_{p}$ is the rest mass of the test particle and $u^{\mu}=$ $d y_{p}^{\mu} / d \tau$ its four velocity. The point particle stress slightly disturbs the background geometry $\bar{g}_{\mu \nu}, \bar{f}_{\mu \nu}$ (the theory has two metrics) describing the $\mathrm{BH}$ and a graviton of mass $\mu$. The latter is given by a specific combination of the coupling parameters of the theory [9]. Here, we study backgrounds for which the two metrics $\bar{g}_{\mu \nu}$ and $\bar{f}_{\mu \nu}$ are proportional, leading to geometries which coincide with those of GR [18] [see the Supplemental Material [12] and Refs. [5,9,18-21]].

The stress energy tensor contributes with fluctuations $\left(\delta g_{\mu \nu}, \delta f_{\mu \nu}\right)$, which we analyze in tensor spherical harmonics and Fourier decompose. Technical details are left for the Supplemental Material [12].

Head-on collisions: Hereafter, we consider two prototypical dynamical processes: head-on collisions and pure equatorial motion corresponding to quasicircular inspirals (once radiation reaction is taken into account). The complete expressions for the source components are shown in the Supplemental Material [12]. For radial motion, axial perturbations are not excited. The multipolar expansion describes only polar-type perturbations with $\ell \geq 0$. Of these, the $\ell \geq 2$ equations contain small $\mu$-dependent corrections to the GR expressions. We do not consider these any further [such corrections were studied in some detail in the weak-field, slow-motion limit elsewhere [10] ] and focus on the truly unique properties of massive gravity: the presence of new degrees of freedom, described by the $\ell=0$ and $\ell=1$ modes.

For the monopole, $\ell=0$ mode, the number of perturbation functions reduces to the four metric components $\left(H_{0}, H_{1}, H_{2}, K\right)$ [see the Supplemental Material [12] and Ref. [9]]. Through the following transformation

$$
K=\frac{\sqrt{-4 \mu^{2} M+\mu^{4} r^{3}+2 \mu^{2} r+4 r \omega^{2}}}{r^{5 / 2}} \varphi_{0},
$$

we obtain a single wave equation for $\varphi_{0}$

$$
\frac{d^{2} \varphi_{0}}{d r_{\star}^{2}}+\left[\omega^{2}-V_{\mathrm{pol}}^{\ell=0}(r, \omega)\right] \varphi_{0}=\mathcal{S}_{\mathrm{pol}}^{\ell=0} .
$$

Here, $V_{\mathrm{pol}}^{\ell=0}(r, \omega)$ is a radial potential whose expression is lengthy and not very illuminating, whereas $r_{\star}$ is a tortoise coordinate defined by $d r_{\star} / d r=1 / f$. The potential $V_{\mathrm{pol}}^{\ell=0}(r, \omega) \sim \mu^{2}$ at large spatial distances, and it vanishes close to the BH horizon. The source term $\mathcal{S}_{\mathrm{pol}}^{\ell=0}$ depends on the radial position and on the point particle's energy. In the highly relativistic regime

$$
\mathcal{S}_{\mathrm{pol}}^{\ell=0}=\frac{8 \sqrt{2} m_{p} \gamma(r-2 M)\left(\mu^{2} r+2 i \omega\right) e^{i \omega t_{p}(r)}}{\sqrt{r}\left(-4 \mu^{2} M+\mu^{4} r^{3}+2 \mu^{2} r+4 r \omega^{2}\right)^{3 / 2}},
$$

where $\gamma$ is the Lorentz boost factor of the test particle at large spatial separations. Here, the $z$ axis is chosen to coincide with the particle trajectory; hence only $m=0$ modes are excited.

For the dipole $\ell=1$ term, the perturbations are completely determined by two coupled equations for $K$ and $\eta_{1}$, which can be recast in a linear form as

$$
\left(\frac{d}{d r_{\star}}+V_{\mathrm{pol}}^{\ell=1}(r)\right) \Sigma=\mathcal{S}_{\mathrm{pol}}^{\ell=1},
$$

where $\Sigma=\left(K, \eta_{1}, d K / d r_{\star}, d \eta_{1} / d r_{\star}\right) T$, and $V_{\text {pol }}^{(1)}$ is a $4 \times 4$ matrix which is shown within the Supplemental Material [12]. For a radial infalling particle with a relativistic boost factor, the source vector is simply given by

$$
\mathcal{S}_{\mathrm{pol}}^{\ell=1}=\left(0,0, S_{K}, S_{\eta_{1}}\right)=(0,0, f(r) / r, 1) S_{\eta_{1}},
$$

where $f(r)=(1-2 M / r)$ and

$$
S_{\eta_{1}}=-\frac{8 \sqrt{6} m_{p} \gamma\left(2+r^{2} \mu^{2}+2 i r \omega\right) e^{i \omega t_{p}(r)}}{4 M r^{2} \mu^{2}-8 M-6 r^{3} \mu^{2}-r^{5} \mu^{4}-4 r^{3} \omega^{2}} .
$$

Quasicircular inspirals: For circular motion, the only nontrivial new degree of freedom is the dipolar-polar component. Our system of equations can be written as

$$
\begin{gathered}
K^{\prime \prime}+a_{1} K^{\prime}+a_{2} K+a_{3} \eta_{1}^{\prime}+a_{4} \eta_{1}=S_{1} \delta\left(r-r_{p}\right), \\
\eta_{1}^{\prime \prime}+b_{1} \eta_{1}^{\prime}+b_{2} \eta_{1}+b_{3} K^{\prime}+b_{4} K=S_{2} \delta\left(r-r_{p}\right),
\end{gathered}
$$

where primes stand for tortoise derivatives, and $r_{p}$ is the orbital radius of the test particle. The system above can be cast in the form

$$
\left[\frac{d}{d r_{\star}}+V_{\mathrm{pol}}^{\ell=1}(r)\right] \Sigma=\mathcal{S}_{\mathrm{circ}}^{\ell=1}
$$

being $\quad \Sigma=\left(K, \eta_{1}, d K / d r_{\star}, d \eta_{1} / d r_{\star}\right)^{T} \quad$ and $\quad \mathcal{S}_{\text {circ }}^{\ell=1}=$ $\left(0,0, S_{K}^{\text {circ }}, S_{\eta_{1}}^{\text {circ }}\right)$. We solve Eq. (10) by first constructing a $4 \times 4$ fundamental matrix $X$ built with the homogeneous solution of the previous system, which yields the general solution

$$
\Sigma(\omega, r)=X \int_{-\infty}^{\infty} X^{-1} \mathcal{S}_{\text {circ }}^{\ell=1} d r_{\star}
$$

Note that the source vector contains linear combinations of the Dirac delta and its derivative. Therefore, integrating by parts Eq. (11), we can immediately obtain an explicit form for the metric functions, $\Sigma(\omega, r)=X[\mathbf{A}+\mathbf{B}]$, where $\mathbf{A}$ and $\mathbf{B}$ are two vectors given by 


$$
\begin{gathered}
\mathbf{A}=\left(1-\frac{2 M}{r_{p}}\right) X^{-1}\left(r_{p}\right) \mathcal{S}_{\text {circ }}^{\ell=1}\left(r_{p}\right), \\
\mathbf{B}=-\frac{d}{d r}\left[\left(1-\frac{2 M}{r}\right) X^{-1} \mathcal{S}_{\text {circ }}^{\ell=1}\right]_{r=r_{p}} .
\end{gathered}
$$

Numerical results.-In this section, we describe the numerical results obtained by solving the systems of ordinary differential equations for the monopole and dipole components of the polar sector. As described in the previous section, we consider circular and radial trajectories: for both the configurations, axial modes are not excited, as the source terms vanish. We integrate Eqs. (3) and (5) through a Green function approach, with appropriate boundary conditions at the $\mathrm{BH}$ horizon and at spatial infinity [see the Supplemental Material [12] for further details].

Head-on collisions: For head-on collisions, the waveform amplitude scales linearly with the mass of the infalling point particle, and the only free parameter is the relative velocity at large distances. We fix this to be relativistic, and we find, as expected, that the amplitude then scales linearly with the boost factor $\gamma$. Although our formalism includes the general case, relativistic collisions should mimic well the late stages of inspiral. In addition, and perhaps more important for us here, they should also describe even explosive events such as supernovae. In theories of massive gravity, even spherically symmetric explosive events release a non-negligible amount of radiation in the monopole mode.

The energy spectrum $d E / d \omega$ for the monopole perturbation is shown in Fig. 1 as a function of the frequency $\omega$, for a head-on collision. The spectrum peaks close to the value of the graviton mass and quickly decays to zero for higher frequencies. The total integrated energy is not shown but it scales like $E_{\mathrm{tot}} \sim 0.01 \mu \mathrm{m}_{p}^{2} \gamma^{2}$ at small couplings $M \mu$.

Knowing the solution in the frequency domain, we can immediately compute the GW signal as a function of the retarded time by simply applying a Fourier transform to

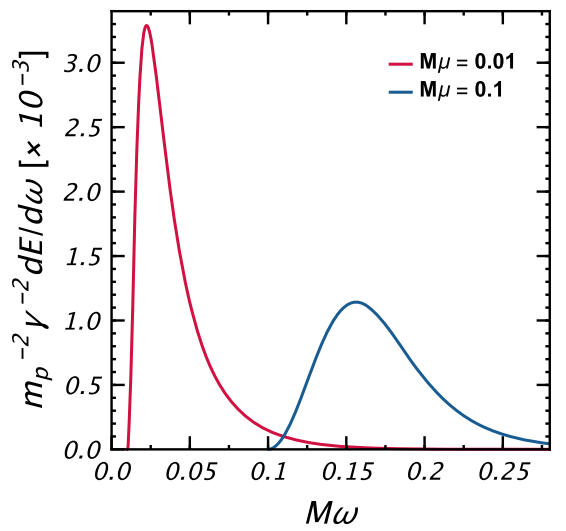

FIG. 1. GW energy spectrum $d E / d \omega$ for the $\ell=0$ polar mode, with $M \mu=(0.1,0.01)$ and a radial infalling particle. $\varphi_{0}(\omega)$. This is shown in Fig. 2 for two values of the extraction radii $R=r \mu=(10,100)$ [22]. It is important to highlight that GWs in theories of massive gravity are dispersed: the waveform at large distances is no longer a function only of $t-r$. This property is apparent in Fig. 2 and was also recently discussed in other setups [23]. We find that the peak of the time-domain waveform can be described by the following scaling

$$
\varphi_{0}^{\text {peak }} \sim \kappa \frac{m_{p} \gamma M^{2}}{(M \mu)^{5 / 2} R^{1 / 2}},
$$

where $\kappa \simeq 0.055$, when the extraction radius $R>1$. This is not too surprising, given the $\mu$ dependence of the source term, Eq. (7), at low frequencies $\omega \sim \mu$. Our results indicate that the delay between the peak of the amplitude and the early signal can be approximated by the following law

$$
\begin{aligned}
(t-r)^{\mathrm{peak}} & \sim(M \mu)^{-0.34} M R \\
& \sim 1800\left(\frac{M}{M_{\odot}}\right)^{0.66}\left(\frac{\mu}{10^{-23} \mathrm{eV}}\right)^{0.66} \frac{r}{8 \mathrm{kpc}} \mathrm{sec} .
\end{aligned}
$$
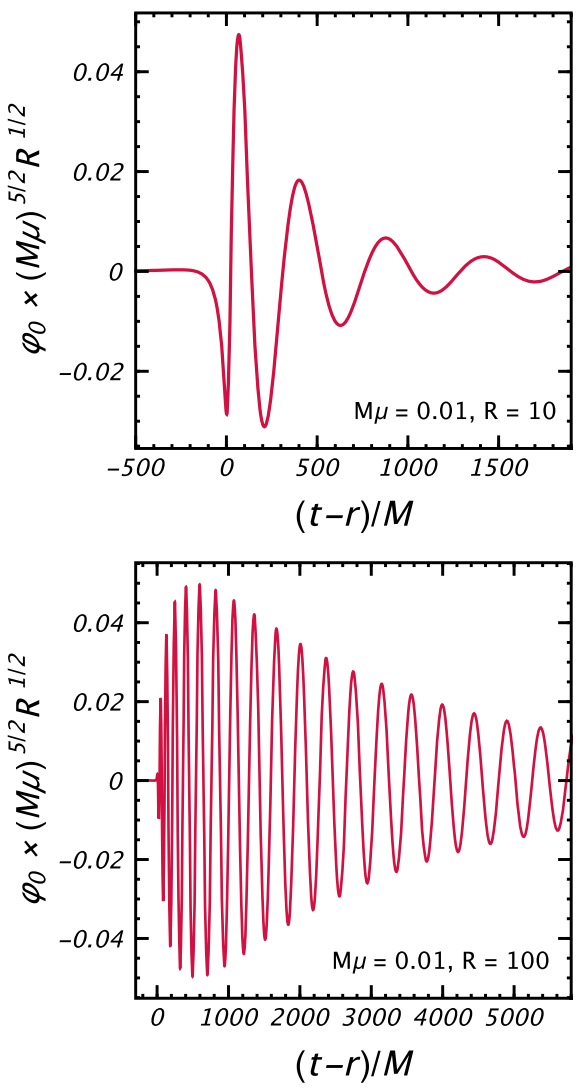

FIG. 2. Gravitational waveforms for the $\ell=0$ component of the polar sector, and a radial infalling particle, as a function of the retarded time $(t-r) / M$. We consider $M \mu=0.01$ and different extraction radius at $R=(10,100)$. The waveform scales trivially with the $\mathrm{BH}$ mass $M$ and the particle mass and boost $m_{p}, \gamma$, according to Eq. (14). 
When expressed in terms of physical metric perturbations, we find

$$
\begin{aligned}
K^{\text {peak }} & =\kappa \frac{m_{p}}{M}\left(\frac{M}{r}\right)^{3 / 2} \frac{1}{M \mu} \\
& \sim 10^{-16} \frac{m_{p} \gamma}{0.01 M} \sqrt{\frac{M}{M_{\odot}}}\left(\frac{8 \mathrm{kpc}}{r}\right)^{3 / 2} \frac{10^{-23} \mathrm{eV}}{\mu} \\
& \sim 10^{-22} \frac{m_{p} \gamma}{0.01 M} \sqrt{\frac{M}{M_{\odot}}}\left(\frac{\mathrm{Gpc}}{r}\right)^{3 / 2} \frac{10^{-25} \mathrm{eV}}{\mu}
\end{aligned}
$$

These numbers are encouraging; however the large-amplitude signals carry a low-frequency content $\omega \sim \mu$, corresponding to a frequency [24]

$$
f \sim 2.5 \times 10^{-9}\left(\frac{\mu}{10^{-23} \mathrm{eV}}\right) \mathrm{Hz} .
$$

Thus, observations of these signals will require lowfrequency sensitive detectors.

At late times and large extraction radii, the waveform is exponentially damped. We cannot rule out power-law decay at very late times. We have searched for the characteristic ringdown modes in this theory and find both good agreement with previously reported values [9] and with the ones inferred from the time-domain waveforms. We note in particular the presence of an unstable mode, which does not seem to be significantly excited on these timescales. Waveforms for the $\ell=1$ mode are shown in Fig. 3 (again for relativistic collisions). The maximum

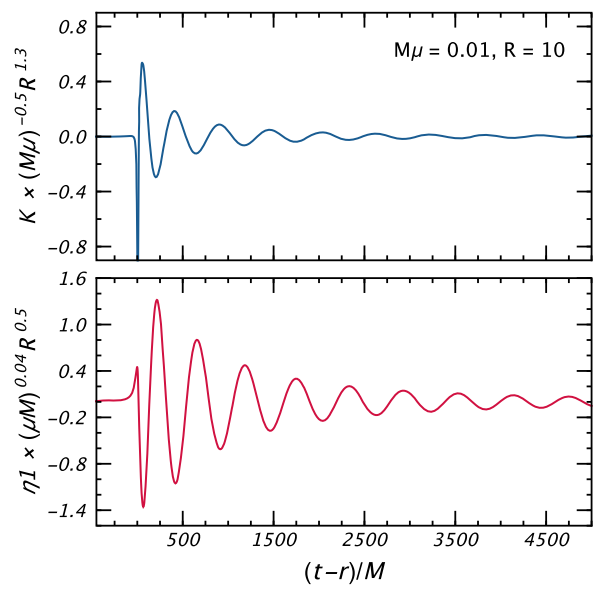

FIG. 3. Waveforms obtained for the $\ell=1$ polar mode, derived for a radial infalling particle with source term given by Eq. (7), as a function of the retarded time $(t-r) / M$. The panel refers to $M \mu=0.01$ at extraction radii $R \mu=10$. The overall behavior is the same as the monopole $\ell=0$ mode. The maximum amplitudes of the two metric functions scale as $K^{\text {peak }} \sim m_{p} \gamma \delta_{1} \sqrt{M \mu} / R^{1.3}$ and $\eta_{1}^{\text {peak }} \sim m_{p} \gamma M \delta_{2}(M \mu)^{-0.04} / R^{1 / 2}$ where $\left(\delta_{1}, \delta_{2}\right) \simeq(0.84,1.3)$ (these expressions also provide the scaling with $\left.M, m_{p}, \gamma\right)$. value of the amplitudes can be described again with a scaling factor of the form given by Eq. (13) (see caption of Fig. 3).

Our results can also be applied to spherically symmetric collapse: in such a case, the source term is trivially replaced by a spherically symmetric shell; the final source term is unchanged. Even if 1\% of the star's rest mass is involved in the collapse, our results indicate that the peak waveform is detectable when $\mu$ is small enough. In fact, Eq. (14) implies that stronger constraints can be obtained via (non-) observations of GWs from collapsing stars in our galaxy. Such conclusions are consistent also with recent results of corecollapse supernovae in massive scalar-tensor theories of gravity [23].

Particles in circular motion: Quasicircular inspirals in the weak-field, slow-motion approximation have been used to impose constraints on massive theories of gravity using pulsar timing observations [10]. Those constraints used only corrections - which scaled like $\mu^{2}$ - to the quadrupole formula. Our results include relativistic motion in stronggravity situations. In GR, particles in circular motion excite only quadrupolar or higher modes. As we saw, a new, dipolar mode arises in massive gravity, the energy flux of which is shown in Fig. 4.

For a particle in a circular orbit of radius $r_{p}$, our results indicate that the flux in the $\ell=m=1$ mode scales like $1 / r_{p}^{4}$, so truly a dipolar behavior, with $\mathrm{BHs}$ having a nontrivial dipolar charge in this theory. Furthermore, the charge is non-negligible at small $M \mu$. We find a flux $d E / d t \sim 0.6 m_{p}^{2} M^{2} / r_{p}^{4}$ [25]. On the other hand, the quadrupole formula in GR predicts a quadrupolar emission $d E / d t=(32 / 5) m_{p}^{2} M^{3} / r_{p}^{5}$. This is one of our main results: the dipolar emission in massive gravity theories dominates the GR quadrupolar term, at arbitrarily small $\mu$. Thus, observations of binary $\mathrm{BHs}$ can potentially be used to rule out these theories [26]. We are extrapolating point-particle results to BH spacetimes. Such procedure was shown to be

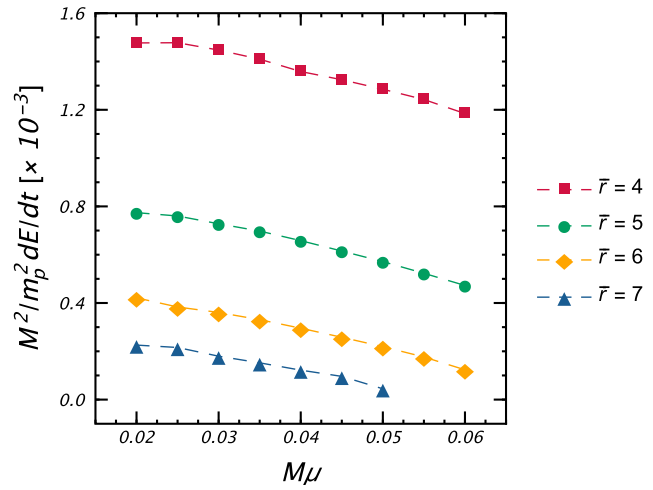

FIG. 4. GW luminosity $d E / d t$ for the $(\ell, m)=(1,1)$ polar mode as a function of the 2-spin field mass $M \mu$ for different radius $\bar{r}=r_{p} / M$ of the test particle on circular orbits around the $\mathrm{BH}$. 
robust in GR when the interacting objects are both BHs [27-29]. When stars are involved, interference effects decrease the total energy output $[30,31]$.

Discussion.-We have worked out the details of gravitational radiation in theories of massive gravity, when two BHs merge. It is clear that a substantial fraction of the radiation emitted in this process decays slowly, at large distances. In fact, because the graviton is massive, lowenergy GWs are confined [see also Ref. [23]]. Such radiation will clearly have an impact in any star or object located within its sphere of influence, but such effects are unknown to us so far. Circular motion at an orbital frequency $\omega=\mu$ will likely lead to resonant excitations of dipolar GWs. Unfortunately, the numerical study of such resonances is a challenging task [32-34], upon which we did not embark.

Technically, our procedure is free of computational challenges. The perturbative framework that we use is an expansion in mass ratio. All the observables that we extract are finite and tend to be zero when the mass ratio decreases. Thus, perturbation theory is applicable and never breaks down as long as mass ratios are sufficiently small (in a welldefined manner). The numerical results are convergent and show that new modes are excited to a substantial amplitude, both in head-on collisions and in quasicircular motion. For head-on collisions-because new modes are excited at characteristically small frequencies-GW detectors sensitive to low-frequency radiation will be able to impose constraints on the mass of gravitons tighter than ever before. In fact, if we trust that our results carry over to two, nearly equal-mass neutron stars, then the constraints on the mass of the graviton will be improved by 2 orders of magnitude or more. The dipolar mode excited by quasicircular inspirals is in fact dominant with respect to the GR quadrupolar emission. Thus, accurate observations of binary BHs have the potential to tightly constraint massive gravity.

Alternatively, our results can be a manifestation that the background geometry does not describe astrophysical BHs. Indeed, Schwarzschild (and Kerr) BHs are unstable in theories with a massive graviton $[9,35,36]$. Nevertheless, for small mass coupling $M \mu$ the instability timescale is extremely large and the spacetime responds to short-timescale phenomena "unaware" of the instability. Thus, sufficiently short-scale phenomena are expected to produce Schwarzschild BHs, and our methods and results apply in the regime where we would like them to, that of small graviton masses. In addition, numerical results suggest that when one of the metrics is taken to be nondynamical, hairy stationary BHs do not even exist [36,37]. One cannot exclude the possibility that a viable astrophysical $\mathrm{BH}$ is described by a dynamical metric [38], in which case our results could change considerably. In particular, Vainshtein screening may play a critical role in more generic background $\mathrm{BH}$ solutions [25]. Notwithstanding, it is clear that
GW astronomy carries a huge potential to understand theories of massive gravity: the existence of extra degrees of freedom lead in general to substantially different dynamics and GW emission. To fully realize this potential, several challenges (including the correct description of astrophysical BHs) need to be seriously tackled.

We are indebted to Evgeny Babichev, Claudia de Rham, Chris Moore, Andrew Tolley, and Luis Lehner for many and very useful comments, discussions, and suggestions. The authors acknowledge financial support provided under the European Union's H2020 ERC Consolidator Grant "Matter and strong-field gravity: New frontiers in Einstein's theory" Grant Agreement No. MaGRaTh646597. This project has received funding from the European Union's Horizon 2020 research and innovation programme under the Marie Sklodowska-Curie Grant Agreement No. 690904. We acknowledge financial support provided by FCT/Portugal through Grant No. PTDC/MATAPL/30043/2017. We acknowledge the SDSC Comet and TACC Stampede2 clusters through NSF-XSEDE Grant No. PHY-090003. The authors would like to acknowledge networking support by the GWverse COST Action CA16104, "Black holes, gravitational waves and fundamental physics."

vitor.cardoso@tecnico.ulisboa.pt

gcabritac@gmail.com

andrea.maselli@tecnico.ulisboa.pt

[1] E. Berti et al., Classical Quantum Gravity 32, 243001 (2015).

[2] L. Barack et al., arXiv:1806.05195.

[3] K. Hinterbichler, Rev. Mod. Phys. 84, 671 (2012).

[4] C. de Rham, Living Rev. Relativity 17, 7 (2014).

[5] S. F. Hassan and R. A. Rosen, J. High Energy Phys. 02 (2012) 126.

[6] C. M. Will, Phys. Rev. D 57, 2061 (1998).

[7] B. P. Abbott et al. (Virgo and LIGO Scientific Collaborations), Phys. Rev. Lett. 116, 221101 (2016); 121, 129902(E) (2018).

[8] B. P. Abbott et al. (Virgo and LIGO Scientific Collaborations), Phys. Rev. Lett. 119, 141101 (2017).

[9] R. Brito, V. Cardoso, and P. Pani, Phys. Rev. D 88, 023514 (2013).

[10] L. S. Finn and P. J. Sutton, Phys. Rev. D 65, 044022 (2002).

[11] E. Kilicarslan and B. Tekin, arXiv:1805.02240.

[12] See Supplemental Material at http://link.aps.org/ supplemental/10.1103/PhysRevLett.121.251103, which includes Refs. [10,13-16], for technical details about analytical and numerical methods used to derive and integrate tensor perturbations in massive gravity theories.

[13] T. Regge and J. A. Wheeler, Phys. Rev. 108, 1063 (1957).

[14] F. J. Zerilli, Phys. Rev. D 2, 2141 (1970).

[15] N. Sago, H. Nakano, and M. Sasaki, Phys. Rev. D 67, 104017 (2003).

[16] P. Pani, V. Cardoso, and L. Gualtieri, Phys. Rev. D 83, 104048 (2011). 
[17] V. Cardoso, L. Gualtieri, C. Herdeiro, and U. Sperhake, Living Rev. Relativity 18, 1 (2015).

[18] S. F. Hassan, A. Schmidt-May, and M. von Strauss, J. High Energy Phys. 05 (2013) 086.

[19] C. de Rham, G. Gabadadze, and A. J. Tolley, Phys. Rev. Lett. 106, 231101 (2011).

[20] V. Baccetti, P. Martin-Moruno, and M. Visser, Classical Quantum Gravity 30, 015004 (2013).

[21] C. de Rham and G. Gabadadze, Phys. Rev. D 82, 044020 (2010).

[22] We checked that the results scale very simply with extraction radius for equal to or larger than these. In fact, we made careful comparisons with the scaling for a simple massive scalar field. Our results are consistent and agree also with the results of Ref. [23]. We believe we are in the asymptotic regime where nonlinearities are not relevant.

[23] U. Sperhake, C. J. Moore, R. Rosca, M. Agathos, D. Gerosa, and C. D. Ott, Phys. Rev. Lett. 119, 201103 (2017).

[24] R. Brito, V. Cardoso, C. F. B. Macedo, H. Okawa, and C. Palenzuela, Phys. Rev. D 93, 044045 (2016).

[25] One might worry that our results are not the GR limit when the mass of the graviton vanishes, and that a "Vainshtein suppression" of the extra modes is not active. We note, however, that there is no support to the claim that if there is a Vainshtein mechanism for stars, then there is one for black holes. Having a Vainshtein mechanism depends both on a theory and a (background) solution. In other words, it is incorrect to relate GR solutions in bigravity theories to the expectation of Vainshtein suppression for perturbations. We thank Evgeny Babichev for discussions on this issue.

[26] E. Barausse, N. Yunes, and K. Chamberlain, Phys. Rev. Lett. 116, 241104 (2016).

[27] E. Berti, V. Cardoso, T. Hinderer, M. Lemos, F. Pretorius, U. Sperhake, and N. Yunes, Phys. Rev. D 81, 104048 (2010).

[28] A. Le Tiec, Int. J. Mod. Phys. D 23, 1430022 (2014).

[29] U. Sperhake, V. Cardoso, C. D. Ott, E. Schnetter, and H. Witek, Phys. Rev. D 84, 084038 (2011).

[30] M. P. Haugan, S. L. Shapiro, and I. Wasserman, Astrophys. J. 257, 283 (1982).

[31] L. I. Petrich, S. L. Shapiro, and I. Wasserman, Astrophys. J. 58, 297 (1985).

[32] V. Cardoso, S. Chakrabarti, P. Pani, E. Berti, and L. Gualtieri, Phys. Rev. Lett. 107, 241101 (2011).

[33] N. Yunes, P. Pani, and V. Cardoso, Phys. Rev. D 85, 102003 (2012).

[34] R. Fujita and V. Cardoso, Phys. Rev. D 95, 044016 (2017).

[35] E. Babichev and A. Fabbri, Classical Quantum Gravity 30, 152001 (2013).

[36] R. Brito, V. Cardoso, and P. Pani, Phys. Rev. D 88, 064006 (2013).

[37] M. S. Volkov, in Proceedings of 14th Marcel Grossmann Meeting on Recent Developments in Theoretical and Experimental General Relativity, Astrophysics, and Relativistic Field Theories (MG14) (In 4 Volumes), Rome, Italy, 2015 (World Scientific, 2017), Vol. 2, pp. 1779-1798, doi: 10.1142/9789813226609_0184.

[38] R. A. Rosen, J. High Energy Phys. 10 (2017) 206. 